\title{
ASSESSMENT OF LIVER FIBROSIS USING REAL-TIME SHEAR-WAVE ELASTOGRAPHY FOR PATIENTS WITH HEPATITIS B E ANTIGEN-NEGATIVE CHRONIC HEPATITIS B AND ALANINE TRANSAMINASE $<2$ TIMES THE UPPER LIMIT OF NORMAL
}

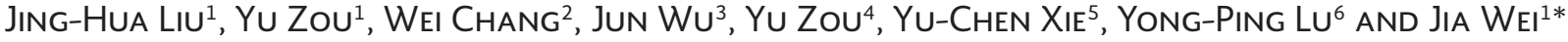 \\ Departments of ${ }^{1}$ Gastroenterology and Hepatology, the 4th Affiliated Hospital of Kunming Medical University; \\ ${ }^{2}$ Biostatistics, Kunming Medical University; ${ }^{3}$ Neurosurgery, Kunming City First People's Hospital; ${ }^{4}$ Hepatology, \\ ${ }^{5}$ Pathology and ${ }^{6}$ Diagnostic Imaging, the 4th Affiliated Hospital of Kunming Medical University. Kunming, China
}

\begin{abstract}
Background: We assessed liver fibrosis using real-time shear-wave elastography (SWE) combined with liver biopsy (LB) for patients with hepatitis $B$ e antigen ( $\mathrm{HBeAg}$ )-negative chronic hepatitis $\mathrm{B}(\mathrm{CHB})$ and alanine transaminase $<2$ times the upper limit of normal and hepatitis B virus DNA < $2000 \mathrm{lU} / \mathrm{ml}$. Methods: A total of 107 patients met the inclusion criteria. Real-time SWE and ultrasoundassisted liver biopsies were consecutively performed. Fibrosis was staged according to the METAVIR scoring system. Analyses of receiver operating characteristic curve were performed to calculate the optimal area under the receiver operating characteristic curve for F0-F1 versus F2-F4, F0-F2 versus F3-F4, and F0-F3 versus F4 for real-time SWE. Results: The most concurrent liver fibrosis degrees were between $\mathrm{F} 1$ and F2 for these $\mathrm{HBeAg}$-negative $\mathrm{CHB}$ patients. Liver stiffness increased in parallel with the degree of liver fibrosis using SWE measurements. The area under the receiver operating characteristic curves was 0.881 ( $95 \%$ confidence interval [Cl]: 0.704-1.000) for SWE ( $p=0.004) ; 0.912$ (95\% Cl: 0.836-0.987) for SWE ( $p=0.000) ; 0.981$ ( $95 \%$ Cl: 0.956-1.000) for SWE $(p=0.000) ; 0.974(95 \% \mathrm{Cl}: 0.936-1.000)$ for SWE ( $=0.000)$ when comparing F0 versus F1-F4, F0-F1 versus F2-F4, F0-F2 versus F3-F4, and FO-F3 versus F4, respectively. Conclusions: SWE has the advantage of providing an image of liver stiffness in real-time. As an alternative to LB, the development of all these noninvasive methods for dynamic evaluation of liver fibrosis will decrease the need for LB, making clinical care safer and more convenient for patients with liver diseases.
\end{abstract}

Key words: Alanine transaminase. Chronic hepatitis B. Elastography. Liver biopsy. Liver fibrosis. Ultrasound.

\section{INTRODUCTION}

Chronic hepatitis B virus (HBV) infection (CHB) is a major liver disease ${ }^{1}$. Patients with high HBV DNA levels need antiviral therapy, while those with low viral loads may not need it. However, the patients with low viral loads, mildly elevated, but with severe liver fibrosis are still at risk of developing hepatocellular carcinoma (HCC); thus, antiviral therapy is indicated in these patients. Therefore, it is important to effectively determine the liver fibrosis stage in patients with low viral loads².

*Corresponding author:

Jia Wei

Department of Gastroenterology and Hepatology

The $4^{\text {th }}$ Affiliated Hospital

Kunming Medical University

Kunming, China

E-mail:weijiaahkmu@yeah.net
Liver biopsy (LB) is considered the gold standard method to stage liver disease and to assess the degree of fibrosis but it is not widely used in resource-limited settings because of its high cost, invasiveness, patient discomfort, risk of complications, sampling error, as well as the need for expert histological interpretation ${ }^{3}$.

Several noninvasive fibrosis tests based on blood or serum indices (aspartate aminotransferase [AST]to-platelet ratio index [APRI], FIB-4 and a commercial assay - FibroTest ${ }^{\circ}$ ) or those with ultrasound principles

Received for publication: 16-03-2017

Approved for publication: 30-05-2017

doi: $10.24875 / R I C .17002215$ 
(transient elastography [TE], e.g., FibroScan ${ }^{\circ}$ ) are now available and increasingly used for evaluating and staging liver fibrosis, which reduces the need for LB in persons with an established cause of liver disease. The use of accurate and validated noninvasive tests in resource-limited settings could help with the optimal selection of persons with $\mathrm{CHB}$ for antiviral therapy. The WHO recommended APRI assessment as a noninvasive diagnostic method for cirrhosis in adults in regions of limited resources ${ }^{4}$. The index was previously reported to be useful in liver fibrosis diagnosis 5 .

In addition, various noninvasive imaging methods for evaluating liver fibrosis have rapidly improved as alternatives to LB. Liver fibrosis has been predicted by $T E^{4,6}$. Ultrasound elastography provides objective data on tissue elasticity by representing the stiffness of tissue using grayscale or color images ${ }^{7,8}$. Real-time shear-wave elastography (SWE) was developed on this basis; this method has attracted increasing attention because it can rapidly, noninvasively, objectively, and quantitatively detect the degree of fibrosis in patients with liver disease, including chronic hepatitis $\mathrm{C}(\mathrm{CHC})^{8}$. Representative reports of noninvasive imaging methods such as SWE for evaluating liver fibrosis, however, have included patients with cirrhosis that have high elasticity values, e.g., receiver-operator curve $(\mathrm{ROC})$ analysis for fibrosis stages F0-F1 versus F2-F4, or F0-F2 versus F3-F49,10.

In this study, we evaluated the diagnostic impact of SWE in hepatitis $B$ e antigen ( $\mathrm{HBeAg}$ )-negative $\mathrm{CHB}$ patients with alanine aminotransferase (ALT) < 2 times the upper limit of normal (ULN) and HBV DNA $<2,000 \mathrm{lU} / \mathrm{ml}$, most of whom had liver fibrosis but did not reach the point of cirrhosis. In particular, we analyzed the accuracy of SWE for distinguishing between nonsignificant (FO-F1) and significant (F2-F3) liver fibrosis. In addition, we compared its ability to diagnose liver fibrosis with APRI.

\section{MATERIALS AND METHODS}

\section{Study patients}

For 2 years (2014-2016), a multi-sectional study was carried out for the assessment of liver fibrosis using real-time SWE for patients with $\mathrm{HBeAg}$-negative $\mathrm{CHB}$ and ALT $<2$ times the ULN (the normal range of ALT is $0-40 \mathrm{U} / \mathrm{I}$ ). A total of 111 patients were hospitalized, confirmed with $\mathrm{CHB}$, and scheduled for LB at the Department of Gastroenterology and Hepatology of the Fourth Affiliated Hospital of Kunming Medical University. All the patients met the following inclusion criteria: (i) HBeAg-negative, (ii) HBV DNA $<2,000 \mathrm{IU} / \mathrm{ml}$, (iii) ALT $<2$ times the ULN, and (iv) without other causes of liver disease (alcoholic liver disease, other liver viral infection, autoimmune liver disease, metabolic liver disease, drug-induced hepatitis, fatty liver, or liver cancer). The diagnostic criterion for $\mathrm{CHB}$ was a positive hepatitis B surface antigen persisting for more than 6 months. Before $\mathrm{LB}$, patient characteristics, epidemiological data, and biochemical tests were recorded. All patients underwent LB. All patients had been asked about a family history of HCC, and those who gave a positive answer were excluded from this study because antiviral therapy was still recommended even if ALT was $<2$ times the ULN. The study protocol was approved by the Institutional Ethics Committee. All the participants gave their informed written consent. This study was not sponsored by the manufacturer of the real-time SWE.

APRI is a simple index for estimating hepatic fibrosis based on a formula derived from AST and platelet concentrations and can be expressed as:

APRI $=*($ AST/ULN $) \times 100) /$ platelet count $\left(10^{9} /\right)^{5}$.

\section{Real-time shear-wave ultrasound elastography of the liver}

Real-time SWE studies were performed using the Aixplorer $^{\mathrm{TM}}$ ultrasound system (SuperSonic Imagine, S.A., Aix-en-Provence, France) with a convex broadband probe (frequency of $3.5 \sim 5.0 \mathrm{MHz}$ ). In SWE, shear waves are created in tissue from the acoustic radiation force generated by focalized ultrasound pulses. A series of these push pulses creates plane shear waves, which propagate over a region of tissue. The speed of the shear wave is then estimated by a Doppler-like acquisition over a region ${ }^{11}$. Finally, this shear wave speed can be used to calculate the tissue stiffness by the formula $E=\rho c^{2}$, where $E$ is tissue elasticity $(\mathrm{kPa}), \rho$ the tissue density $\left(\mathrm{kg} / \mathrm{m}^{3}\right)$, and $\mathrm{c}$ the shear wave velocity $(\mathrm{m} / \mathrm{s})$. The elasticity estimates are then color-coded, creating a 2D quantitative SWE image $(\mathrm{kPa})$ of tissue stiffness, which is displayed in box form over a conventional B-mode image. The size and position of the SWE image are user-adjustable, enabling a trade-off in frame rate and extent of view. 
By placing a circular region of interest (ROI) in a SWE image, the mean and standard deviation of the elasticity within the ROI can be displayed. In this study, we used a SWE box size of $3 \mathrm{~cm}$.

The SWE measurements were performed on the right lobe of the liver, through intercostal spaces with the patient lying in the supine position with the right arm in maximal abduction. The same intercostal space was used for both SWE measurements, which was successively performed. The upper edge of the SWE box was placed 1.5-2.0 cm from Glisson's capsule in the liver and an area of parenchyma free of large vessels. This placement of SWE box aided in avoiding reverberation artifacts beneath Glisson's capsule and pulsations around larger vessels, both of which could lead to erroneously elevated shear wave speeds. Measurements of liver stiffness were obtained from the average of a circular ROI, $2 \mathrm{~cm}$ in diameter when scanning conditions permitted. The circular ROI was reduced in diameter if limitations in viable signal within the SWE box prohibited a $2 \mathrm{~cm}$ diameter. Measurements were classified as failed when no/little signal was obtained in the SWE box for all the acquisitions. The mean value of four consecutive measurements was used for statistical analyses. Due to temporal persistence, the displayed SWE image and measurements were the result of roughly three averaged frames in time. The entire real-time SWE examination lasted approximately 5 minutes per patient.

Our SWE measurements have been checked for their reproducibility in assessing liver elasticity in 225 healthy volunteers ${ }^{12}$. These healthy subjects showed real-time SWE values ranging from $5.79 \pm 1.33 \mathrm{kPa}$ (95\% confidence interval: 5.66-5.92).

\section{LB and histopathological analysis}

Ultrasound-assisted percutaneous LB was performed by two experienced physicians (Y.Z. and J.H.L.) using an intercostal approach. The same intercostal space, which was used for SWE measurements, was used for the LB. A disposable, $1.6 \mathrm{~mm}$ in diameter, modified Magnum needle (Magnum ${ }^{\circledR}$, Bard ${ }^{\circledR}$, US) was used. All biopsy specimens were fixed in formalin and embedded in paraffin. The length of each LB specimen (in millimeters) was recorded.

The specimens were read on site by a single expert liver pathologist (Y.C.X.), blind to the results of real-time
SWE, but not to the patient's clinical and biochemical data. Liver fibrosis and necroinflammatory activity were evaluated semi-quantitatively according to the METAVIR scoring system ${ }^{13}$. Fibrosis was staged on a five-point scale from 0 to 4 according to the METAVIR scoring system (FO, absent; F1, enlarged fibrotic portal tract; F2, periportal or initial portal-portal septa but intact architecture; F3, architectural distortion but no obvious cirrhosis; $F 4$, cirrhosis $)^{13}$. Necroinflammatory activity was graded as follows: $A 0=$ none, $A 1=$ mild, $\mathrm{A} 2=$ moderate , and $\mathrm{A} 3=$ severe $^{8}$.

\section{Statistical analysis}

Descriptive statistics were produced for demographic, clinical, and laboratory characteristics for this study sample of patients. The Shapiro-Wilk test was used to test the normal distribution of quantitative variables. When quantitative variables were normally distributed, the results were expressed as mean values and standard deviation (SD); otherwise, median and interquartile range (IQR; $25^{\text {th }}-75^{\text {th }}$ percentile) were reported. Qualitative variables were summarized as counts and percentages. Pearson rank coefficient was used to test the correlation between two study variables. Quantile regression was used for a multivariate model to evaluate the association between real-time SWE and fibrosis, APRI, necroinflammation, and biochemical tests. Kruskal-Wallis one-way analysis of variance by ranks was used to test the measurement differences with real-time SWE among different fibrosis stages. A frequency distribution was obtained for choosing optimal cutoff values of real-time SWE to maximize the sum of sensitivity and specificity for different fibrosis thresholds: FO-F1 versus F2-F4 (F > 2), FO-F2 versus $F 3-F 4(F>3)$, and $F 0-F 3$ versus $F 4(F=4)$. The diagnostic performance of real-time SWE and their combinations was assessed using ROC and the area under ROC (AUROC) analysis.

Comparisons of AUROCs were done using the method described by DeLong et al. for correlated data $^{14}$. Agreement between fibrosis stages and real-time SWE was illustrated in a contingency table and weighted $\mathrm{K}$ was carried out. Data analysis was performed with SPSS 20.0 statistical package and Medcalc v16.2.0.

The study was conducted and written according to the Standards for Reporting of Diagnostic Accuracy ${ }^{15}$. 


\section{RESULTS}

In all, 111 patients met the inclusion criteria. In two patients, liver stiffness measurements failed with real-time SWE due to narrow intercostal spaces in one patient and due to obesity (body mass index $>32 \mathrm{~kg} / \mathrm{m}^{2}$ ) in the other patients. There were 70 men and 41 women, with an average age of 39.42 years. The characteristics of the 111 patients are summarized in Table 1. The mean length of $L B$ specimens was $27 \mathrm{~mm}$ (SD: $8 \mathrm{~mm}$; range: 10-55 mm); the length was $>15 \mathrm{~mm}$ in 103/111 (92.7\%) cases and > $25 \mathrm{~mm}$ in $65 / 111(58.6 \%)$ cases. In univariate analysis, realtime SWE showed a high correlation to the degree of fibrosis. The corresponding values for real-time SWE were (i) for liver fibrosis: $r=0.805\left(p<10^{-5}\right)$, (ii) for the degree of necroinflammation: $r=0.27(p=0.004)$, and (iii) for HBV DNA: $r=0.198(p=0.037)$. No correlation with other variables was found. Multivariate regression analysis, including METAVIR stage, METAVIR grade, and APRI, confirmed the correlation for SWE with fibrosis stage $(p<0.001)$, but not with all other

Table 1. Patients' demographics and their biochemical and histological data by liver biopsy examination

\begin{tabular}{|c|c|}
\hline Characteristics & $\mathrm{n}=111$ \\
\hline Sex (men) & $70(63.1 \%)$ \\
\hline Age $(y)$ & $\begin{array}{l}39.42 \text { (SD: } 7.945 ; \\
\text { range: } 15-60)^{*}\end{array}$ \\
\hline BMI $\left(\mathrm{kg} / \mathrm{m}^{2}\right)$ & $\begin{array}{c}23.78 \text { (SD: } 3.641 ; \\
\text { range: } 17.80-33.10)^{*}\end{array}$ \\
\hline ALT & $\begin{array}{l}34.00(\mathrm{IQR}: 25-46 \\
\text { range: } 12-69)^{\dagger}\end{array}$ \\
\hline AST & $\begin{array}{l}37.00(\text { IQR: } 27-43 ; \\
\text { range: } 9-79)^{*}\end{array}$ \\
\hline HBV DNA & $\begin{array}{c}259.00(\text { IQR: } 10-530 ; \\
\text { range: } 2-1,256)^{\dagger}\end{array}$ \\
\hline APRI & $\begin{array}{c}0.55 \text { (IQR: } 0.40-0.71 ; \\
\text { range: } 0.12-1.18)^{\dagger}\end{array}$ \\
\hline SWE & $\begin{array}{l}9.13(\mathrm{SD}: 1.405 \\
\text { range: } 5.57-12.20)^{*}\end{array}$ \\
\hline \multicolumn{2}{|c|}{ Fibrosis score (METAVIR) } \\
\hline FO-F1 & $38(34.2 \%)$ \\
\hline $\mathrm{F} 2$ & $53(47.7 \%)$ \\
\hline F3 & $15(13.5 \%)$ \\
\hline $\mathrm{F} 4$ & $5(4.5 \%)$ \\
\hline \multicolumn{2}{|c|}{ Activity grade (METAVIR) } \\
\hline AO & $1(0.9 \%)$ \\
\hline $\mathrm{A} 1$ & $30(27.0 \%)$ \\
\hline A2 & $68(61.3 \%)$ \\
\hline $\mathrm{A} 3$ & $12(10.8 \%)$ \\
\hline
\end{tabular}

${ }^{*}$ Mean value and SD; ${ }^{\dagger}$ Median value and IQR.

ALT: alanine aminotransferase; APRI: AST-to-platelet ratio index;

AST: aspartate aminotransferase; BMI: body mass index;

IQR: interquartile range; SD: standard deviation;

SWE: shear-wave elastography. variables. Within each fibrosis stage, no correlation was found between METAVIR activity grade and SWE measurements (Fig. 1).

Median values, IQR, range, number of outliers, and $\mathrm{p}$ values of measurements obtained for each fibrosis stage with SWE are shown in Table 2. Figs. 2-5 show ROCs for significant and severe fibrosis, as well as cirrhosis, and improvements in AUROCs by real-time SWE for each level of fibrosis. For significant fibrosis $(F \geq 2)$, a statistically significant improvement $(p<0.001)$ in the AUROCs was observed in real-time SWE (0.912). The slight improvements for the AUROCs for severe fibrosis and cirrhosis were not significant $(p=0.000$ and $p=0.000$, respectively).

The optimal cutoff values for the different levels of fibrosis were determined by analysis of ROCs for realtime SWE. Sensitivity, specificity, AUROCs, positive predictive value, negative predictive value, positive

Figure 1. Box-and-whisker plots of shear-wave elastography values for each METAVIR stage in relation to the degree of necroinflammatory activity. Liver stiffness values are reported on the $y$-axis, and METAVIR grade of necroinflammatory activity on the $x$-axis. The central box represents values from the lower to upper quartile (25-75 percentile). The line through each box represents the median. Error bars show minimum and maximum nonextreme values. A, necroinflammatory activity (METAVIR grade); F, fibrosis (METAVIR stage).

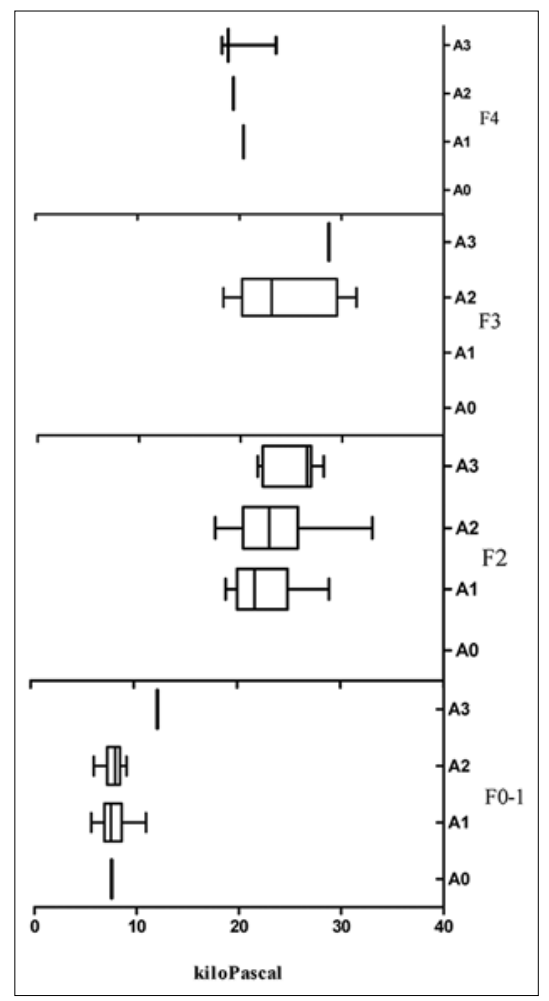


Table 2. Median values, interquartile range, range, outliers, and $p$ values of measurements obtained for each fibrosis stage with shear-wave elastography and transient elastography

\begin{tabular}{llccccc}
\hline METAVIR stage & Method & F0 & F1 & F2 & F3 & F4 \\
\hline Mean & SWE & 6.97 & 7.97 & 9.34 & 10.83 & 11.63 \\
SD & SWE & 1.390 & 1.224 & 0.510 & 0.362 & 0.556 \\
Range & SWE & $5.57-9.30$ & $5.83-12.03$ & $7.40-10.60$ & $10.37-11.50$ & $10.77-12.20$ \\
Number of outliers & SWE & 0 & 0 & 0 & 0 & 0 \\
p value & & & $0.013^{*}$ & $<0.001^{\dagger}$ & $<0.001^{\ddagger}$ & $0.064^{\S}$ \\
\hline
\end{tabular}

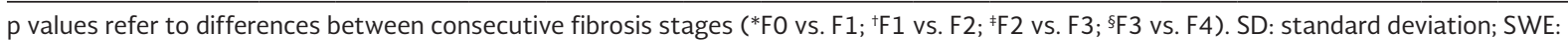
shear-wave elastography

Figure 2. Receiver operating characteristic curves for real-time shear-wave elastography for different fibrosis thresholds: (a) F0-F1 versus F2-F4 ( F > 2), (b) F0-F2 versus F3-F4 ( F > 3), (c) FO-F3 versus $\mathrm{F} 4(\mathrm{~F}=4) .95 \%$ confidence intervals $(\mathrm{Cls})$ are shown in parentheses. Area under the receiver operating characteristic curve, 6.82 , was 0.881 (95\% Cl; 0.805-0.934; $\mathrm{p}=0.004)$. SWE: shear-wave elastography.

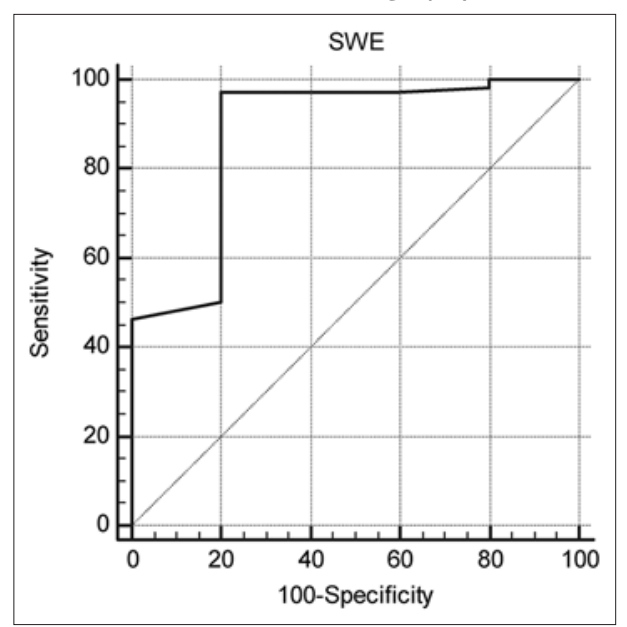

likelihood ratio, and negative likelihood ratio of optimal cutoff values for each METAVIR stage are shown in Table 3. Real-time SWE showed a high sensitivity and specificity in assessing significant $(F>2)$ and advanced $(F>3)$ fibrosis.

Table 4 shows the concordance rate of real-time SWE versus METAVIR stage. Overall, real-time SWE correctly classified 70/111 (63.0\%) patients. Weighted $\mathrm{K}$ was 0.74 . Real-time SWE performed worse in $\mathrm{F} 4$ stage, probably due to the small number of cases of liver cirrhosis.

\section{DISCUSSION}

In this study, the diagnostic accuracy of real-time SWE in estimating liver fibrosis was compared against histology in $\mathrm{HBeAg}$-negative $\mathrm{CHB}$ patients with ALT $<2$ times the ULN and HBV DNA $<2000 \mathrm{IU} / \mathrm{ml}$. The AUROCs in differentiating no/mild fibrosis (FO-F1)
Figure 3. Receiver operating characteristic curves for shearwave elastography for different fibrosis thresholds: (a) FO-F1 versus F2-F4 ( F > 2), (b) F0-F2 versus F3-F4 (F > 3), (c) F0-F3 versus $F 4(F=4) .95 \%$ confidence intervals $(C l s)$ are shown in parentheses. Area under the receiver operating characteristic curve, 8.62, was 0.912 (95\% Cl: 0.836-0.987; $\mathrm{p}=0.000$ ). SWE: shear-wave elastography.

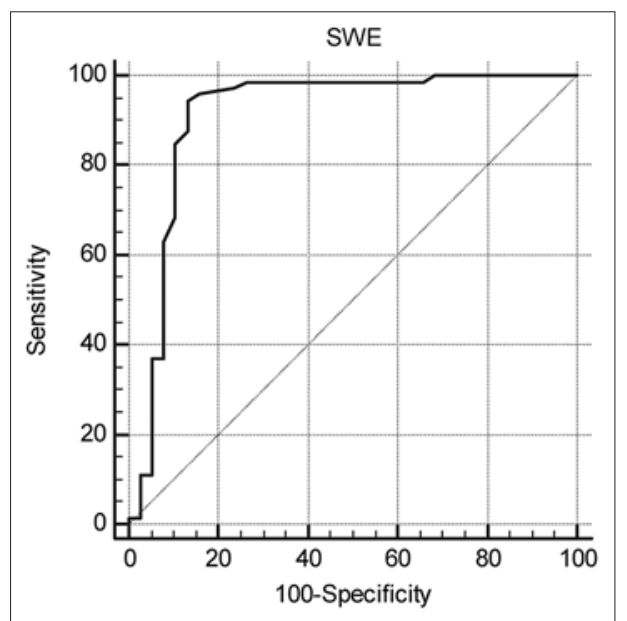

Figure 4. Receiver operating characteristic curves for real-time shear-wave elastography for different fibrosis thresholds: (a) F0-F1 versus F2- F4 ( $>>2$ ), (B) F0-F2 versus F3-F4 $(F>3),(C) F 0-F 3$ versus $F 4(F=4) .95 \%$ confidence intervals (Cls) are shown in parentheses. Area under the receiver operating characteristic curve, 10.30, was 0.981 (95\% Cl: 0.935-0.997; $p=0.000$ ). SWE: shear-wave elastography.

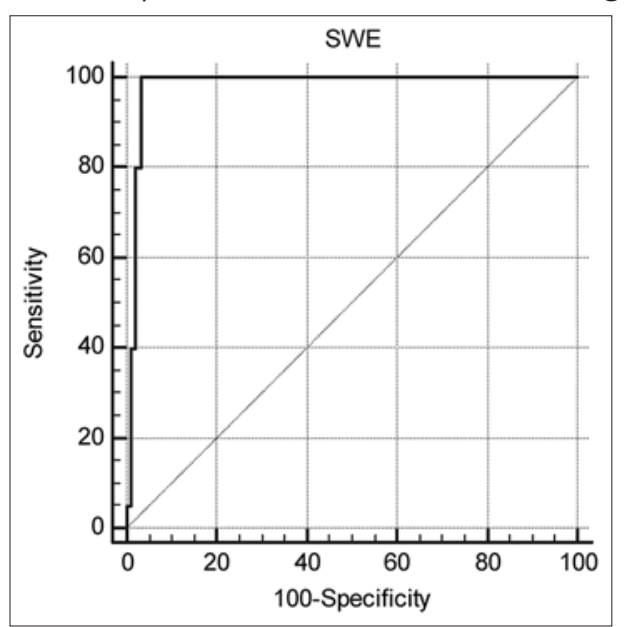


from significant fibrosis ( $F>2$ ) were 0.912 for realtime SWE $(p=0.000)$. The performance of real-time SWE in identifying significant fibrosis $(F \geq 2)$ and severe fibrosis ( $F \geq 3$ ) was quite high. These findings suggest

Figure 5. Receiver operating characteristic curves for real-time shear-wave elastography for different fibrosis thresholds: (a) FO-F1 versus F2-F4 (F> 2), (b) FO-F2 versus F3-F4 (F > 3), (C) FO-F3 versus $\mathrm{F} 4(\mathrm{~F}=4)$. $95 \%$ confidence intervals $(\mathrm{Cls})$ are shown in parentheses. Area under the receiver operating characteristic curve, 10.72, was 0.974 (95\% Cl: 0.924-0.995; $p=0.000$ ). SWE: shear-wave elastography.

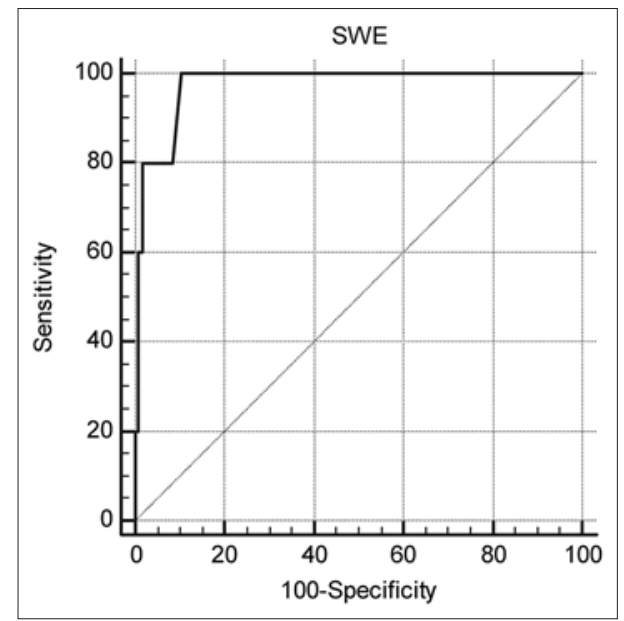

Table 3. Resulting clinical performance of shear-wave elastography using optimal measurements cutoff values

\begin{tabular}{lccccc}
\hline Parameter & Method & F $\geq$ e1 & F et2 & F $\geq$ e3 & F = 4 \\
\hline Cutoff in kPa SWE & 6.82 & 8.62 & 10.30 & 10.72 \\
Sensitivity, \% SWE & 97.2 & 94.5 & 100.0 & 100.0 \\
Specificity, \% SWE & 80.0 & 86.8 & 96.7 & 89.6 \\
PPV, \% & SWE & 99.0 & 93.2 & 87.0 & 31.3 \\
NPV, \% & SWE & 57.1 & 89.2 & 100.0 & 100.0 \\
LR+ & SWE & 4.86 & 7.18 & 30.3 & 9.64 \\
LR- & SWE & 0.04 & 0.06 & - & - \\
\hline
\end{tabular}

kPa: kilopascal; LR+: positive likelihood ratio; LR-: negative likelihood ratio; NPV: negative predictive value; PPV: positive predictive value;

SWE: shear-wave elastography. that real-time SWE can be used for the assessment of significant fibrosis, severe fibrosis, and cirrhosis.

To the best of our knowledge, this is the first study aimed at the noninvasive assessment of liver fibrosis by real-time SWE measurements in $\mathrm{HBeAg-negative}$ CHB patients with ALT $<2$ times the ULN and HBV DNA $<2000 \mathrm{IU} / \mathrm{ml}$ undergoing LB on the same day. Tada et al. estimated the utility of real-time SWE for assessing $\mathrm{CHC}$ infection without cirrhosis comparing with liver fibrosis indices, but not with liver histology and not in CHB patients. A prototype system was used where a SWE image was retrospectively generated. The authors report similar SWE values for the lower fibrosis stages, but slightly higher values for F3 and F4, along with larger IQRs per stage, than those found in our series. The extra targeting information from the real-time SWE image combined with a smaller sampling region of liver parenchyma could explain the differences between our results at higher stages of fibrosis. The higher stages of fibrosis have been shown to have greater spatial heterogeneity.

Noninvasive assessment of liver disease stage at baseline and during follow-up. Blood tests such as APRI are recommended as the preferred noninvasive test to assess for the presence of cirrhosis (APRI score $>2$ in adults) in resource-limited settings ${ }^{5}$. In our study, the average of APRI score was 0.55 (Fig. 1); no patient had a score of 2, although many patients had significant or advanced fibrosis. That is because all included patients had mild ALT elevation and their platelets were in the normal range so APRI could not assess the presence of cirrhosis in these patients.

Sonoelastography (SE) appears to be a reliable and fast modality for noninvasive assessment of liver fibrosis in patients with chronic viral hepatitis and other liver

Table 4. Analysis of concordance of shear-wave elastography versus METAVIR stage

\begin{tabular}{|c|c|c|c|c|c|c|}
\hline \multirow[t]{2}{*}{ Young's modulus } & \multicolumn{4}{|c|}{ METAVIR } & \multirow[t]{2}{*}{ Total } & \multirow[t]{2}{*}{ Concordance rate $(\%)$} \\
\hline & F0-F1 & F2 & F3 & $\mathrm{F} 4$ & & \\
\hline \multicolumn{7}{|l|}{ F0-F1 } \\
\hline $\begin{array}{l}\text { SWE WE8.62 } \\
\text { F2 }\end{array}$ & 33 & 4 & 0 & 0 & 37 & 89.2 \\
\hline $\begin{array}{l}8.62<\text { SWE WE10.30 } \\
\text { F3 }\end{array}$ & 3 & 48 & 0 & 0 & 51 & 94.1 \\
\hline $\begin{array}{l}10.30<\text { SWE WE10.72 } \\
\text { F4 }\end{array}$ & 0 & 1 & 6 & 0 & 7 & 85.7 \\
\hline \multicolumn{7}{|l|}{ Cumulative concordance } \\
\hline SWE & & Weigh & 0.74 & & $70 / 111$ & 82.9 \\
\hline
\end{tabular}

kPa: kilopascal; SWE: shear-wave elastography. 
diseases $^{16}$. Among various SE methods, TE was introduced first and has been tested in the largest number of patients ${ }^{17}$. However, because it is mono-dimensional, TE cannot be used for ultrasound examination, nor is it applicable for patients with ascites ${ }^{18}$. Supersonic SWE is the first among ultrasound elastography methods allowing for the quantitative analysis of liver stiffness in real time ${ }^{16,19}$. It is, therefore, possible to visually control the quality of elastography maps, which may increase the reliability of liver stiffness measurement as compared to other SE methods. On the other hand, the ability to visually control the elasticity maps in real time and to choose the "proper" one for liver stiffness measurement could be criticized as a potential source of subjectivity. The reliability of real-time two-dimensional SWE for the estimation of liver fibrosis severity appears to be comparable to other SE methods, with somewhat better performance reported for the early stages of liver fibrosis $9,10,20-24$.

In this study, the correlation between fibrosis stage data and liver stiffness values assessed with real-time SWE was not affected by necroinflammation, which is in agreement with previous studies assessing liver elasticity with TE or magnetic resonance ${ }^{25,26}$. In other studies in which an influence of necroinflammatory activity on TE measurements has been found ${ }^{27,28}$, the inclusion criteria were different from those applied to our study. In fact, in their series, patients with hepatitis $B$ and other etiologies were also included ${ }^{29}$. On the other hand, all patients in our series had ALT values $<2$ times the ULN.

In our series, SWE correctly classified $82.9 \%$ of patients. Even though LB is still considered the benchmark for validation of noninvasive techniques aimed at assessing the degree of liver fibrosis, its accuracy is challenged by sampling errors and intra- and interobserver variability ${ }^{30,31}$. Moreover, the METAVIR scoring system does not take into account quantitative changes in liver collagen but is largely an assessment of architectural changes in a small sample of the liver $^{32}$. While the distribution of fibrosis in the liver is heterogeneous, the histological staging is based on a biopsy specimen that represents at most $1 / 50,000$ of the total liver mass ${ }^{33}$.

The management and prognosis of $\mathrm{CHB}$ strongly rely on the degree of liver fibrosis. In patients with $\mathrm{CHB}$, the assessment of the stage of fibrosis is a crucial issue before therapy. Treatment should be initiated promptly in patients with the severity of necroinflammation (METAVIR score $A \geq 2$ ) and fibrosis (METAVIR score $F \geq 2)^{1,2}$. In this study, real-time SWE improved the ability to identify significant fibrosis in comparison with LB. The recent guidelines for the management of hepatitis $B$ infection of the American Association for the study of liver diseases and WHO have indicated that noninvasive methods can now be used instead of LB in patients with $C H B$ to assess liver disease severity before therapy at a safe level of predictability ${ }^{1}$.

According to currently available guidelines, patients with $\mathrm{HBeAg}$-negative $\mathrm{CHB}$ and $\mathrm{ALT}<2$ times ULN should be closely followed up, and those with ALT $>2$ times ULN should be given antiviral therapy. However, these patients may have undergone early or obvious liver fibrosis at normal or low ALT levels and should be given antiviral therapy as soon as possible. A traditional biopsy is invasive and may easily cause complications, so not all patients accept this procedure. In contrast, non-invasive liver fibrosis assessment may be prone to detect hepatic inflammatory activities, and the patients with normal or low ALT levels may be less sensitive to this method. Therefore, we recommend performing both examinations for these patients to compare the correlation.

This study has limitations. First, our series of patients had a variable distribution of the different stages of fibrosis, particularly for F2. Second, our study did not take into account the FIB-4 index and the Forns index as indices of liver fibrosis. Third, the analysis was carried out in a relatively small number of patients and it would be critical to validate these results in larger studies.

In summary, we made the assessment of liver fibrosis using real-time SWE combined with LB for patients with $\mathrm{HBeAg}$-negative $\mathrm{CHB}$ and $\mathrm{ALT}<2$ times the ULN and HBV DNA < $2000 \mathrm{IU} / \mathrm{ml}$ in 111 cases. The results of this study show that real-time SWE was as accurate as $L B$ in assessing significant fibrosis $(F \geq 2)$ in $\mathrm{HBeAg}$-negative $\mathrm{CHB}$ patients with $\mathrm{ALT}<2$ times the ULN and HBV DNA $<2000 \mathrm{IU} / \mathrm{ml}$. SWE has the advantage of producing images of liver stiffness in real time, while guided by a B-mode image. As a result, liver stiffness measurements benefit from the guidance of both anatomical and tissue stiffness information. As an alternative to $L B$, the development of all these noninvasive methods for dynamic evaluation of liver fibrosis will decrease the need for LB, making clinical 
care safer and more convenient for patients with liver diseases. Further studies in larger patient populations are needed to confirm these results and the values of the thresholds of real-time SWE for the different fibrosis stages.

\section{REFERENCES}

1. WHO Guidelines for the Prevention, Care and Treatment of Persons with Chronic Hepatitis B Infection. Available from: http:// www.who.int/hiv/pub/hepatitis/hepatitis-b-guidelines/en. [Last accessed on 2015 Mar 12]

2. Terrault NA, Bzowej NH, Chang KM, Hwang JP, Jonas MM, Murad $\mathrm{MH}$; American Association for the Study of Liver Diseases. AASLD guidelines for treatment of chronic hepatitis B. Hepatology. 2016;63:261-83.

3. Castéra L, Nègre I, Samii K, Buffet C. Pain experienced during percutaneous liver biopsy. Hepatology. 1999;30:1529-30.

4. Castéra L, Vergniol J, Foucher J, Le Bail B, Chanteloup E, Haaser M, et al. Prospective comparison of transient elastography, Fibrotest, APRI, and liver biopsy for the assessment of fibrosis in chronic hepatitis C. Gastroenterology. 2005;128:343-50.

5. Wai CT, Greenson JK, Fontana RJ, Kalbfleisch JD, Marrero JA, Conjeevaram HS, et al. A simple noninvasive index can predict both significant fibrosis and cirrhosis in patients with chronic hepatitis C. Hepatology. 2003;38:518-26.

6. Ziol M, Handra-Luca A, Kettaneh A, Christidis C, Mal F, Kazemi F, et al. Noninvasive assessment of liver fibrosis by measurement of stiffness in patients with chronic hepatitis C. Hepatology. 2005;41:48-54.

7. Bercoff J, Tanter M, Fink M. Supersonic shear imaging: A new technique for soft tissue elasticity mapping. IEEE Trans Ultrason Ferroelectr Freq Control. 2004;51:396-409.

8. Brunt EM, Janney CG, Di Bisceglie AM, Neuschwander-Tetri BA, Bacon BR. Nonalcoholic steatohepatitis: A proposal for grading and staging the histological lesions. Am J Gastroenterol. 1999;94:2467-74.

9. Bavu E, Gennisson JL, Couade M, Bercoff J, Mallet V, Fink M, et al. Noninvasive in vivo liver fibrosis evaluation using supersonic shear imaging: A clinical study on 113 hepatitis $C$ virus patients. Ultrasound Med Biol. 2011;37:1361-73.

10. Ferraioli G, Tinelli C, Dal Bello B, Zicchetti M, Filice G, Filice C; Liver Fibrosis Study Group. Accuracy of real-time shear wave elastography for assessing liver fibrosis in chronic hepatitis C: A pilot study. Hepatology. 2012;56:2125-33

11. Muller M, Gennisson JL, Deffieux T, Tanter M, Fink M. Quantitative viscoelasticity mapping of human liver using supersonic shear imaging: Preliminary in vivo feasibility study. Ultrasound Med Biol. 2009:35:219-29.

12. Yang DL, Wei J, Zhang Y, Tang YY, Qian P, Lu YP. Quantitative analysis of liver tissue stiffness in healthy adults by real-time shear wave elastography. Chin J Ultrasound Med. 2014;30:411-4.

13. Bedossa P, Poynard T. An algorithm for the grading of activity in chronic hepatitis C. The METAVIR Cooperative Study Group. Hepatology. 1996;24:289-93.

14. DeLong ER, DeLong DM, Clarke-Pearson DL. Comparing the areas under two or more correlated receiver operating characteristic curves: A nonparametric approach. Biometrics. 1988;44:837-45.

15. Bossuyt PM, Reitsma JB, Bruns DE, Gatsonis CA, Glasziou PP, Irwig LM, et al. Towards complete and accurate reporting of studies of diagnostic accuracy: The STARD initiative. Stand Rep Diagn Accuracy Clin Chem. 2003;49:1-6.

16. Cosgrove D, Piscaglia F, Bamber J, Bojunga J, Correas JM, $\mathrm{Gilja} \mathrm{OH}$, et al. EFSUMB guidelines and recommendations on the clinical use of ultrasound elastography. Part 2: Clinical applications. Ultraschall Med. 2013;34:238-53.

17. Tsochatzis EA, Gurusamy KS, Ntaoula S, Cholongitas E, Davidson BR, Burroughs AK. Elastography for the diagnosis of severity of fibrosis in chronic liver disease: A meta-analysis of diagnostic accuracy. J Hepatol. 2011;54:650-9.

18. Bamber J, Cosgrove D, Dietrich CF, Fromageau J, Bojunga J, Calliada $F$, et al. EFSUMB guidelines and recommendations on the clinical use of ultrasound elastography. Part 1: Basic principles and technology. Ultraschall Med. 2013;34:169-84.

19. Bota S, Herkner H, Sporea I, Salzl P, Sirli R, Neghina AM, et al. Meta-analysis: ARFI elastography versus transient elastography for the evaluation of liver fibrosis. Liver Int. 2013;33:1138-47.

20. Sporea I, Bota S, Jurchis A, Sirli R. Acoustic radiation force impulse and supersonic shear imaging versus transient elastography for liver fibrosis assessment. Ultrasound Med Biol. 2013; 39:1933-41.

21. Sporea I, Bota S, Gradinaru-Tascau O, Sirli R, Popescu A, Jurchis A. Which are the cut-off values of 2D-Shear Wave Elastography (2D-SWE) liver stiffness measurements predicting different stages of liver fibrosis, considering Transient Elastography (TE) as the reference method? Eur J Radiol. 2014;83:e118-22.

22. Cassinotto C, Lapuyade B, Mouries A, Hiriart JB, Vergniol J, Gaye D, et al. Non-invasive assessment of liver fibrosis with impulse elastography: Comparison of supersonic shear imaging with ARFI and FibroScan ${ }^{\oplus}$. J Hepatol. 2014;61:550-7.

23. Zeng J, Liu GJ, Huang ZP, Zheng J, Wu T, Zheng RQ, et al. Diagnostic accuracy of two-dimensional shear wave elastography for the non-invasive staging of hepatic fibrosis in chronic hepatitis B: A cohort study with internal validation. Eur Radiol. 2014;24:2572-81.

24. Ferraioli G, Tinelli C, Lissandrin R, Zicchetti M, Bernuzzi S, Salvaneschi L, et al. Ultrasound point shear wave elastography assessment of liver and spleen stiffness: Effect of training on repeatability of measurements. Eur Radiol. 2014;24:1283-9.

25. Huwart L, Sempoux C, Vicaut E, Salameh N, Annet L, Danse E, et al. Magnetic resonance elastography for the noninvasive staging of liver fibrosis. Gastroenterology. 2008;135:32-40.

26. Wong VW, Vergniol J, Wong GL, Foucher J, Chan HL, Le Bail B, et al. Diagnosis of fibrosis and cirrhosis using liver stiffness measurement in nonalcoholic fatty liver disease. Hepatology. 2010:51:454-62.

27. Arena U, Vizzutti F, Corti G, Ambu S, Stasi C, Bresci S, et al. Acute viral hepatitis increases liver stiffness values measured by transient elastography. Hepatology. 2008;47:380-4.

28. Coco B, Oliveri F, Maina AM, Ciccorossi P, Sacco R, Colombatto P, et al. Transient elastography: A new surrogate marker of liver fibrosis influenced by major changes of transaminases. J Viral Hepat. 2007;14:360-9.

29. Fraquelli M, Rigamonti C, Casazza G, Conte D, Donato MF, Ronchi G, et al. Reproducibility of transient elastography in the evaluation of liver fibrosis in patients with chronic liver disease. Gut. 2007; 56:968-73.

30. Bedossa $P$, Dargère $D$, Paradis V. Sampling variability of liver fibrosis in chronic hepatitis C. Hepatology. 2003;38:1449-57.

31. Intraobserver and interobserver variations in liver biopsy interpretation in patients with chronic hepatitis $\mathrm{C}$. The French METAVIR Cooperative Study Group. Hepatology. 1994;20:15-20.

32. Standish RA, Cholongitas E, Dhillon A, Burroughs AK, Dhillon AP. An appraisal of the histopathological assessment of liver fibrosis. Gut. 2006:55:569-78.

33. Poynard T, Munteanu M, Imbert-Bismut F, Charlotte F, Thabut D, Le Calvez S, et al. Prospective analysis of discordant results between biochemical markers and biopsy in patients with chronic hepatitis C. Clin Chem. 2004;50:1344-55. 